\title{
Paraneoplastic neuromyelitis optica spectrum disorder diagnosed with mantle cell lymphoma
}

\author{
Samuel SY Wang ${ }^{1}$, BMed, Thomas KH Lau ${ }^{2 *}$, MB, BS \\ ${ }^{1}$ Faculty of Medicine, University of New South Wales, Kensington, Australia \\ ${ }^{2}$ Department of Clinical Oncology, Prince of Wales Hospital, Shatin, Hong Kong \\ *Corresponding author: Ikh195@ha.org.hk
}

Hong Kong Med J 2018;24:637-8

DOI: 10.12809/hkmj187403

Paraneoplastic neurological syndromes (PNS) are well-recognised neurological symptoms that occur at increased frequency in cancer patients. Paraneoplastic neurological syndromes are independent of metastasis, direct tumour infiltration, or known indirect mechanisms such as toxicity, ectopic hormone secretion, or induced coagulopathies. $^{1} \quad$ Paraneoplastic neurological syndromes may affect any part of the nervous system, and are believed to result when an immunologic response is directed against shared antigens that are ectopically expressed by the tumour, but otherwise predominantly expressed by the nervous system (onconeural antigens). ${ }^{1}$ Antibodies can be detected in the serum or cerebrospinal fluid of many, but not all, patients with PNS. ${ }^{2}$ Diagnosing PNS requires identification of the type of neurological syndrome based on neurological signs and symptoms, the detection of well-characterised onconeural antibodies, and the presence of a cancer. ${ }^{3}$

Paraneoplastic neurological syndromes are rare in patients with solid tumours, and probably even rarer among patients with lymphomas. ${ }^{4}$ The predominant types of PNS in lymphomas are paraneoplastic cerebellar degeneration in Hodgkin's lymphoma, and dermatomyositis/polymyositis in both Hodgkin's lymphoma and non-Hodgkin's lymphoma. Other PNS are uncommon and are reported only in isolated case reports and case series. $^{2}$

Neuromyelitis optica spectrum disorder (NMOSD) is an inflammatory disorder of the central nervous system characterised by severe, immune-mediated demyelination, and axonal damage predominantly targeting optic nerves and spinal cord. Traditionally considered a variant of multiple sclerosis, presence of the disease-specific aquaporin-4 antibody, which plays a direct role in the pathogenesis of NMOSD, distinguishes the two entities. $^{5}$

Recently, NMOSD is increasingly recognised as a paraneoplastic disorder especially in men or in patients who present in older age. ${ }^{6}$ Paraneoplastic NMOSD has been reported in a wide variety of tumour histological types but mostly in solid tumours. ${ }^{6}$

We recently encountered a definite case of PNS in a patient who was diagnosed with mantle cell lymphoma (MCL) and shortly afterwards developed neurological symptoms due to NMOSD. An 83-year-old Chinese man presented to Prince of Wales Hospital, Hong Kong with change in bowel habit and per-rectal bleeding since January 2015. An oesophageal-gastro-duodenoscopy showed diffuse gastritis and biopsy confirmed infiltration by MCL. Subsequent colonoscopy also showed involvement of the rectum by MCL. In March 2015, the patient then presented to Alice Ho Miu Ling Nethersole Hospital, Hong Kong with acute-onset left-sided numbness and right-sided hemiparesis. Brain computed tomography was normal. Subsequent magnetic resonance imaging spine revealed a hyperintense signal from C3 to C8 with oedema in the spinal cord without an intramedullary spaceoccupying lesion (Fig a). He was treated with pulse methylprednisolone for the transverse myelitis which showed gradual neurological improvement. A lumbar puncture was then performed which showed normal total protein and glucose, negative white cell and cytology; however, the sample was obtained after one dose of pulse methylprednisolone therapy. Subsequent staging positron emission tomography/ computed tomography was performed and showed hypermetabolic uptakes at the gastric pylorus, rectosigmoid colon, and spleen. Peripheral blood and bone marrow examination confirmed involvements by MCL. In view of the patient's advanced age and borderline performance status, he was treated with rituximab, chlorambucil, and prednisolone combination therapy. After one cycle of treatment, he developed an acute onset of right eye vision loss, while his right hemiparesis showed persistent improvement. Magnetic resonance imaging of the brain and orbit was then performed, which showed right optic neuritis (Fig b). He was treated with another course of pulse methylprednisolone. Serum anti-aquaporin-4 antibody tested positive, and thus neurologists diagnosed NMOSD. He received a total of six courses of rituximab, chlorambucil, and prednisolone combination therapy with gradual 


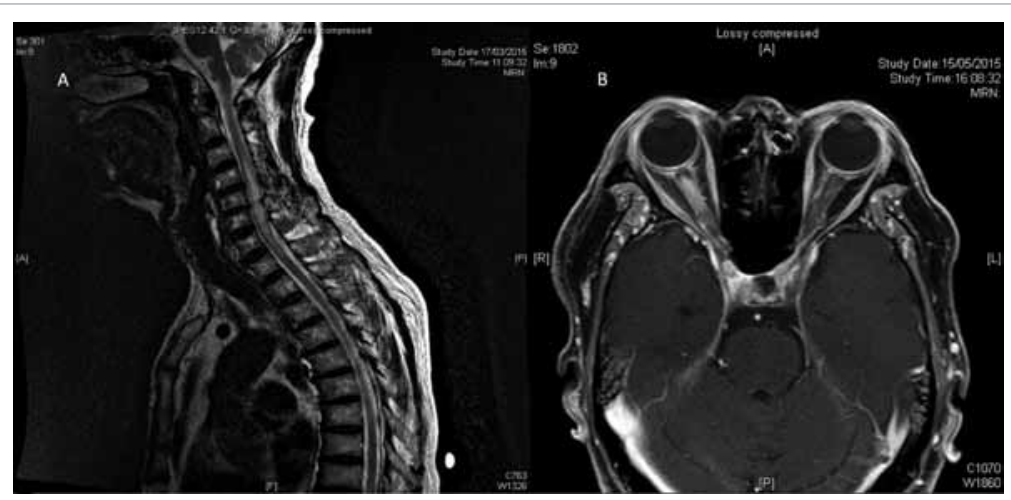

FIG. (a) T2-weighted image of spinal cord magnetic resonance imaging showing oedema from C3 to C8. (b) T2-weighted image of cranial magnetic resonance imaging showing contrast enhancement and a loss of distinction with the optic nerve sheath for patients with NMOSD who present in older age, as opposed to third or fourth decade in idiopathic cases, an underlying malignancy should be suspected. This case also illustrates that effective treatment of the underlying lymphoma is important in controlling neurological disease, though the use of expensive drugs can be challenging in a resourcepoor setting. Our study highlights the importance of the correct diagnosis of PNS. Early recognition of a neurological syndrome as paraneoplastic often leads to the discovery and treatment of the underlying tumour, which is a crucial step in the management of the PNS. ${ }^{1,2}$

\section{Author contributions}

All authors have made substantial contributions to the concept or design; acquisition of data; analysis or interpretation of data; drafting of the article; and critical revision for important intellectual content.

\section{Funding/support}

partial improvement of right eye vision, almost full recovery of limb power, and cessation of per-rectal bleeding. The patient was thus offered maintenance rituximab therapy. The patient remained stable until 16 months later when he developed per-rectal bleeding and palatal ulcers. Biopsy of the palatal ulcers revealed MCL involvement, and MCL therapy was switched to rituximab-cyclophosphamidevincristine-prednisolone for six cycles. The lymphoma progressed on treatment with recurrence of palate nodules, parotid masses, and weakening limb power. Lenalidomide therapy was offered but was not started as the drug was a self-financed item and the patient could not afford it. He was started on maintenance azathioprine for prevention of relapse of NMOSD by the neurologists. However, the patient later developed an acute left vision loss due to left optic neuritis, and sustained a fall injury which resulted in a right hip fracture. The patient became bed-ridden despite hip fracture fixation and was deemed unfit for further anti-cancer therapy. $\mathrm{He}$ was then placed on best supportive care.

Previous reported cases of paraneoplastic NMOSD are mostly associated with solid cancers, most commonly breast and lung cancers. To the best of our knowledge, this is the first reported case of MCL to be associated with paraneoplastic NMOSD. This unusual case supports the suggestion that
This article received no specific grant from any funding agency in the public, commercial, or not-for-profit sectors.

\section{Declaration}

All authors have disclosed no conflicts of interest. All authors had full access to the data, contributed to the study, approved the final version for publication, and take responsibility for its accuracy and integrity.

\section{References}

1. Darnell RB, Posner JB. Oxford, UK: Oxford University Press; 2011. Paraneoplastic syndromes. Contemporary Neurology Series 79: 1-482.

2. Graus F, Ariño H, Dalmau J. Paraneoplastic neurological syndromes in Hodgkin and non-Hodgkin lymphomas. Blood 2014;123:3230-8.

3. Graus F, Delattre JY, Antoine JC, et al. Recommended diagnostic criteria for paraneoplastic neurological syndromes. J Neurol Neurosurg Psychiatry 2004;75:113540 .

4. Giometto B, Grisold W, Vitaliani R, et al. Paraneoplastic neurologic syndrome in the PNS euronetwork database: a European study from 20 centers. Arch Neurol 2010;67:3305.

5. Papadopoulos MC, Verkman AS. Aquaporin 4 and neuromyelitis optica. Lancet Neurol 2012;11:535-44.

6. Sepulveda M, Sola-Valls N, Escudero D, et al. Clinical profile of patients with paraneoplastic neuromyelitis optica spectrum disorder and aquaporin- 4 antibodies. Mult Scler 2017:1352458517731914. 